JOURNAL DE PHYSIQUE IV

Colloque C4, supplément au Journal de Physique III, Volume 5, mai 1995

C4-231

\title{
A Comparison of the Classical and a Modern Theory of Detonation
}

F.E. Walker

Interplay, Danville, California 94526, U.S.A.

ABSTRACT

A quite complete exposition of what has been called the classical theory of detonation is given in the Scientific American of May 1987 by W.C. Davis. However, Davis states in his report that, "In spite of the variety of modern applications of explosives, detonation science has not yet reached maturity . . .," and, "Scientists who study explosions are spurred on by being constantly reminded that the current detonation theory is incomplete." In this paper a comparison is made between the classical theory as expounded by Davis and a more moderm theory based on the concepts that: (1) The energy in the very narrow shock or detonation front is highly nonergodic, and thermal equilibrium, particularly between the translational and vibrational energy modes, does not exist in the front; (2) No realistic temperature can be ascribed to this very narrow zone; and (3) A physical regulator which constrains shock and detonation velocities is directly related to the vibratory velocities of the atoms of the shocked materials.

The paper includes a short historical summary, a statement of some crucial deficiencies in the classical theory, and it contains the presentation and discussion of a number of experiments and mathematical arguments favoring the alternative theory. Among these are experimental observations made in the 1960 s and 1970 s and continuing to the present. Proposals of tribochemical or mechanical bond fracture in shock fronts in explosives were made as early as 1938, and they appeared occasionally in later years, but they were often ignored. Finally, results from more recent experiments and calculations are summarized, which appear to support forcefully the alternative theory. 


\section{INT RODUCTION}

The early history of the study of the detonation of explosives was reviewed by $W . C$. Davis in the May 1987 issue of the Scientific American--from the synthesis and detonation of nitroglycerin by Ascanio Sobrero in 1846 through the development of the ZND theory by Yakov Zel'dovich, John von Neumann, and Werner Doering. He discussed the pioneering analysis done by David Chapman and Emile Jouguet that led to the C-J theory, from which most modern theoretical studies have been derived.

The concepts included in the 2 D model provided useful hypotheses as to how detonation is structured and maintained. According to the model, a shock wave propagates into the unreacted explosive and compresses it instantly. This compression, modeled as a piston moving against the explosive, provides enough heat to initiate chemical reactions (in thermal equilibrium) behind the shock front which release the explosive energy. This chemical energy produces the high temperature and pressure which maintain the detonation. The expansion of the reactions' gases provides the forces that are observed as the useful work, or the destructive power, of the high explosive.

From this theory, a rather complex formalism with the associated mathematics was developed. Davis described this formalism, as shown graphically in Fig. 1. Briefly, a plot of all possible pressure values in a shocked material (the material behind a shock wave) for all possible values of the shock velocity in the material is called a Hugoniot curve (a). All the possible states (pressure and material velocity) of a shocked material for a given shock-wave velocity can be depicted in the Hugoniot-curve coordinate system as a straight line, a Rayleigh line, whose slope is proportional to the shock-wave velocity. The final state of a material under the influence of a shock wave with a given velocity is shown graphically as the point at which its Hugoniot curve intersects the specific Rayleigh line, as seen in Fig. 1(a).

The C-J theory maintains that the point at which the Rayleigh line is tangent to the Hugoniot for the completely reacted explosive (the C-J point) specifies the state from which the reaction products expand to do work. This point also determines the detonation velocity (D) from the slope of the Rayleigh line, as seen in Fig. 1(b). The ZND theory requires Hugoniot curves for the partially reacted explosive, as well

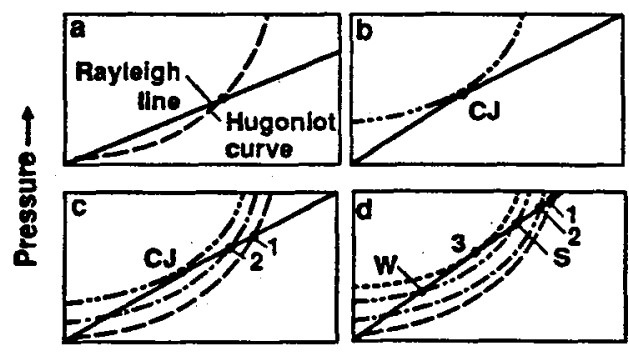

Material velocity $\longrightarrow$

Figure 1. The Hugoniot and Rayleigh curves which represent the $C-J$ and $Z N D$ models. 
as for the unreacted and completely reacted material. The explosive will be at a higher pressure in its unreacted (1) and partially reacted (2) states, as seen in Fig. 1(c). A higher detonation velocity (a steeper Rayleigh line) denotes a "strong" detonation, as seen in Fig. 1(d). In some cases, a temporary state (3) is possible in which the Hugoniot curve lies above the curve for the completely reacted explosive. This state describes a "weak" detonation, which can reach a final state $(W)$ that has a lower material velocity and pressure than in the C-J state.

This is a macroscopic theory that can be modeled with hydrodymamic and thermodynamic algorithms, but it denies the importance or even the necessity for kinetic inputs, and it provides no helpful microscopic insights. As Davis states (Ref. 1), "In spite of the variety of modern applications of explosives, detonation science has not reached maturity . . ., and, "Scientists who study explosions are spurred on by being constantly reminded that the current detonation theory is incomplete."

The C-J and ZND theories lead to the conclusion that the "constant" detonation shock waves observed for particular materials cause the explosive material to turn into gaseous products at a temperature sufficient to just exactly provide the correct pressure to maintain the correct detonation velocity. Therefore, the proponents of these theories have developed a number of equations of state (EOS) with some rather arbitrary coefficients and parameters to calculate the "correct" temperatures and pressures in the reaction products. Two quite recent statements on the failure or inaccuracy of EOSs are given and discussed in a later section.

The principal purpose of this report is to present for comparison a quite different theory, or hypothesis, which describes both initiation and detonation in a microscopic or molecular regime, includes new kinetic principles, and gives a physical explanation for the constancy of detonation velocities.

To direct attention to the significant aspects of the experiments and calculations to be reviewed in the following sections, here are the basic concepts of this modern theory:

1. The initiation of explosive reaction by shock waves in chemical explosives is determined by (a) the production by the the momentum transfer, shear, or energy gradient forces across the shock front of ions, free atoms and radicals, in addition to thermally-activated molecules, randomly distributed within the bulk of the shocked explosive; (b) the growth of reaction sites at the points where sufficient numbers of the free atoms, radicals, ions, and molecular fragments are formed to sustain the appropriate initiation reactions; and (c) the input of a critical quantity of energy fluence from the shock forces to the shock-compressed explosive to enable a minimum number of reaction sites to reach a self-sustaining exothermic reaction. (Ref. 2). 
2. The exceedingly high kinetic energy of momentum transfer in the detonation front is sufficient to cause massive fracture of the covalent bonds of the explosives molecules at and near the front so that the large majority of the molecules are broken, as in the initiating shock, to individual atoms, radicals, molecular fragments, ions, and they are rearranged extensively. These particles can then react in about $10^{-14}$ to $10^{-12} \mathrm{~s}$ to provide the chemical energy which drives the detonation. (Ref. 3 ).

3. The energy in the very narrow shock or detonation front is nonergodic, and thermal equilibrium, particularly between the translational and vibrational energy modes, does not exist in the front. No realistic temperature can be ascribed to this zone (Ref 4).

4. A physical regulator constrains the shock and detonation velocities, and this regulator is directly related to the vibratory velocities of the atoms of the shocked material (Ref. 5). This concept of determining energy release rates or reaction rates through a nonequilibrium process based on the relative vibration velocities of the atom pairs and groups involved, is designated as physical kinetics.

A summary of comparisons of the classical and the modern theory is given in Table 1 to assist in the elucidation of the differences as they are presented in the following discussion.

\section{EARLY SIGNIFICANT CLASSICAL STUDIES}

Discussion of several early experiments and theoretical analyses may aid in understanding the departures of the new theory from what have been called the classical studies in shock initiation and detonation. Campbell et al. (Ref. 6) conducted some elaborate experiments in the early 1960s on the initiation to detonation of homogeneous (nitromethane) and heterogeneous (Ref. 7) (PBX-9404, a plastic-bonded HMX) explosives. Analysis of the experiments in which nitromethane (NM) was initiated with shocks of about $8 \mathrm{GPa}$ and durations of about $1 \mathrm{Hs}$ led the experimenters to the conclusion that the shock wave compressed the NM; the compression heated the NM to some value at which significant thermal reaction began; and, after an induction period, the detonation wave originated at the NM face first impacted, traveled through the compressed liquid overtaking the shock front, and continued into the unshocked material. The entire process was considered to be a thermal equilibrium process. The activation energy presumed was about $59 \mathrm{kcal} / \mathrm{mole}$, as in low temperature thermal decomposition.

The analysis of heterogeneous initiation was not so easily reached, because the bulk temperature in an explosive shocked strongly enough to cause initiation to detonation was believed not to be nearly high enough to produce sufficient 
Table 1. Comparisons of the Classical and the Modern Theories

\section{Classical Theory}

The shock acts as a piston, compression heating, equilibrium thermal decomposition, Arrhenius kinetics, thermodynamic determination and control of detonation velocity, many different equations-of-state required.

No good explanation.

Shock acts as piston for compression heating, requires various concepts of thermal heat-

ing to form hot spots.

No good explanation.

No good explanation, Violation of theory.

No good explanation.

No good explanation.

No good explanation.

No explanation;

violation of theory.

No good explanation.

No good explanation.

Very little help.

No good explanation.

Difficult explanation.

With best equation-of-state, calculation in error by $14.5 \%$
Concepts, Principles and observations

Theory Concept

Eyring 's

Starvation Kinetics

Shock Initiation of Heterogeneous Explosives

Differences in Sensitivity in various Sensitivity Tests

Time to Initiation of NVI at Low Shock Pressures

Acceleration of Shock Front with NonInitiating Shocks

Initiation to Detonation by Free-Radical Gradient

BTNEA Experiment

Increased Detonation Velocity of NM + DETA

Mechanical scission of Bonds in Plastics R. Graham, et al.

Shock-Induced Chemistry, R. Graham et al. Understanding of Microscopic Processes

Isomer Pairs Differ in Thermal or Shock Sensitivity

$$
\text { Detonation }
$$
at Low Velocity

\section{Calculated Detonation} Velocity of E25

(PETN/Paraffin:?5/25)
Modern Theory

Shock energy carried in very narrow zone by momentum transfer, very high energy gradient forces cause mechanical fracture of covalent bonds, physical kinetics, detonation velocity determined and controlled by average relative vibrational velocities of atom pairs or in molecular fragments, equationsof-state not valid.

Corresponds well with mechanical fracture concept and reaction rates observed. Very different from Arrhenius kinetics.

Mechanical fracture of covalent bonds in shock front leads to hot spots, critical energy fluence required for initiation. Explained by different fractions of thermal and shock input.

Explained by physical kinetics.

Mechanical fracture of bonds in and near the shock front.

The high energy gradient forms shock wave which initiates detonation.

Explained by massive mechanical bond scission.

Easily explained by physical kinetics, and it is calculated accurately from Hugoniot data and empirical formulae.

Corroboration of massive bond scission.

Corroboration of massive bond scission.

Provides rational explanations.

It is probable that they would differ, since one decomposition is thermal, and the other is by mechanical bond fracture.

Different kinetic rate due to lower level of bond fracture at lower initiation pressure.

Calculated by Hugoniot values and empirical formula within $0.5 \%$.

Note: There are still questions about how to explain deflagration-to-detonation transfer (DDT) and how to determine the temperature in a shock front. The modern theory proposes temperatures of about 10,000 to $30,000 \mathrm{~K}$ versus 3,000 to $5,000 \mathrm{~K}$ by the thermodynamic theory in the detonation front. 
reaction to lead to a detonation in the time observed. Previously, Bowden, Gurton and Joffe (Refs. 8,9) proposed and observed that small centers of concentrated reaction did occur, and they then assumed that an energy-concentrating mechanism produced "hot spots" in the bulk explosive. Many explanations and processes have been proposed for this phenomenon:

(a) Gases in voids in the explosive were compressed and heated; this heat was transferred to the molecules around the voids; and the reaction started on the void surface.

(b) The shock waves collided or reinforced other waves when they moved through and around the explosives crystals, thus causing spots of higher pressure.

(c) The shock caused friction between the explosive grains, and this friction produced small areas of high temperature.

other explanations were suggested, but none have been well quantified.

To check the concept of the gases in the voids being compressed and heated, experimenters compacted explosives in atmospheres of gases with different heat capacities. The idea was that the gases that were heated to higher temperatures by the shock compression would cause initiation in shorter times. This proposed correlation was not observed.

It has been assumed that there is compression of gases in voids, some friction between grains, and some shock interactions and reflections, but no very convincing arguments have been made that "proved" any of these concepts as the sources of effective hot spots. However, the hypothesis has persisted that some macroscopic process within the shock wave produces hot spots from which the initiating reaction develops.

A number of tests were developed in this earlier period to measure and compare the shock sensitivity of explosives then in use and under study. There were wedge tests, cardgap tests, drop-hammer tests, and gun tests. Analyses of these tests were generally based on a value of shock pressure at which the explosive would detonate, usually some $50 \%$ of the trials. Experimenters observed, but did not explain why, some explosives were more sensitive than others in one test and less sensitive in another. Several tests, such as the drop-hammer and skid tests, had components of heating that resulted from friction or material flow that also led to some confusion in the interpretation of the results, but they did provide useful information for rather specific situations.

A great strength of opinion had developed in the explosives literature and in the community studying initiation and detonation that shock pressure (compression) alone (or predominantly) was the determinant in shock initiation (very likely because of the piston model). As a result of this, 
when a very precise set of some 60 experiments was later performed on the initiation of PBX-9404 (Ref. 10) by the impact of aluminum plates, the experimenter failed to analyze the data properly.

Henry Eyring and colleagues (Ref. 11) at the University of Utah in the $1940 \mathrm{~s}$, studied reaction rates in detonating explosives. One striking result from their observations and analyses in this early period is that the reaction rates, as calculated from the detonation velocities, are quite similar for all of the explosives they studied, even though the lowtemperature decomposition rates and the thermodynamic energy content are quite different. Iater, Eyring and other associates analyzed this disturbing observation again (Ref. 12). They studied materials as different as cyclopropane, tetryl, and mixed explosive compositions. In all cases, they found that the logarithms of the reaction rates for assumed firstorder decompositions were about $6.0 \neq 0.5$ (Ref. 13). Eyring's analysis from reation-rate theory led to his concept of "starvation kinetics," in which he calculated that only 20 degrees of freedom in any of the explosives molecules he studied were contributing energy to the bond that was first to break, no matter how large the molecule might be. This was markedly different from Arrhenius kinetics or a thermal equilibrium process.

\section{SIGNIFICANT EXPERTMENTS AND CALCULATIONS IN SUPPORT OF THE NEW THEORY}

Some 25 years ago, Richard Wasley and I began a series of experiments on the initiation and detonation of explosives with the object of extending the data into some unexplored areas. In preparation for the early experiments, we reviewed some data (Ref. 10) obtained on the shock initiation of PBX9404 with impacting aluminum plates. Our interpretation of the data was very different from that published, and it led to the derivation and publication of the critical energy fluence concept (Ref. 14) of shock initiation for heterogeneous explosives. The equation which describes the concept, $E_{c}=\frac{t p^{2}}{\rho U_{S}}$,

is easily derived from the kinetic energy and Hugoniot equations. It serves well to describe shock initiation in most of the generally-used explosives in the range of shock and detonation pressures from about 0.3 to $40 \mathrm{GPa}$.

In the equation, $t$ is the time width of the initiating shock pulse, $P$ is its pressure, $\rho$ is the initial density of the explosive, and $U_{S}$ is the shock velocity at the pressure $P$.

This concept and the equation provide the explanation for the previously observed sensitivity anomalies in the initiation of a specific explosive in various sensitivity tests. 
Additionally, it was shown (Ref. 15) to provide a rational correlation for data from ten different sources and test methods, over nearly seven orders of magnitude in time and three in pressure, for PBX-9404 and other HMX-based explosives.

The reason this quite simple relationship was not discovered for so many years appears to be a result of the historical development of explosives theories based on the piston concept. Thus pressure, not energy fluence, was considered to be the determinant of initiation. The piston concept was both the genius and the demon of the $\mathrm{C}-J$ and $Z \mathrm{ND}$ theories.

\section{Low-Pressure Initiation of Nitromethane}

The initiation data on nitromethane were meager in 1968. It had been observed that about $8 \mathrm{GPa}$ of shock pressure was required to initiate $N M$ to detonation. Here again, the old concept was held of pressure being the determinant of initiation, but it was found that the detonation was observable in about one microsecond.

In an effort (Ref. 16) to determine if the critical energy hypothesis might also apply in some degree to initiation in homogeneous systems, we used an experimental design (shown in Fig. 2) to provide nearly rectangular shock waves of about 5.0 to $6.5 \mathrm{GPa}$ that persisted for more than 20 microseconds. According to the thermal-equilibrium concept, the time to initiation of $\mathrm{NM}$ at a pressure of $6.0 \mathrm{GPa}$ should be near $0.1 \mathrm{~s}$ (Ref. 6), as seen in Fig. 3. What we observed most clearly in both framing-camera and streak-camera records (see for example Fig. 3) was that initiation at $6.0,6.2$ and $6.5 \mathrm{GPa}$ occurred in approximately 20,16 and $10 \mathrm{microseconds,}$ respectively. This is some 4 orders of magnitude shorter in time (Fig. 3) than predicted by the thermal equilibrium theory.

This very short time to detonation was not the only curious result. It was also evident that the detonation had not started at the nitromethane face first put under pressure and heated, as the old theory required, but it appeared at some position very near the shock front. The film records show clearly that a retonation also proceeds from the initial detonation site back toward the face first impacted. In fact, if the detonation had started at this face first put under compression, the streak-camera film shows that initiation at $6.0 \mathrm{GPa}$ would have occurred in an even shorter time-about 8 microsecpnds.

The criticism from the explosives community was that there must have been some defect in the five very consistent experiments. However, after more than 20 years, no defect has been found or reported. 


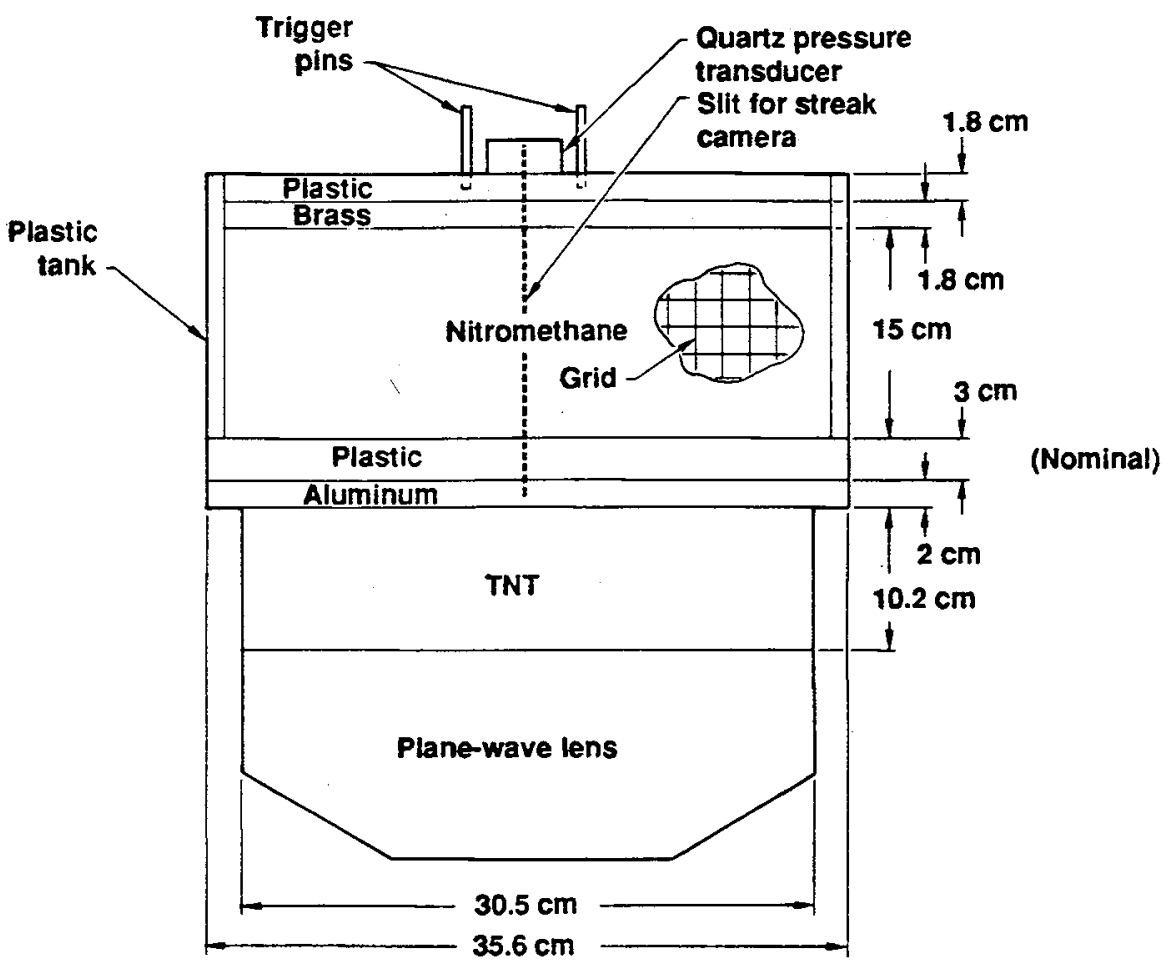

Figure 2. Design of the experiments used to study the lower pressure shock initiation of nitromethane.

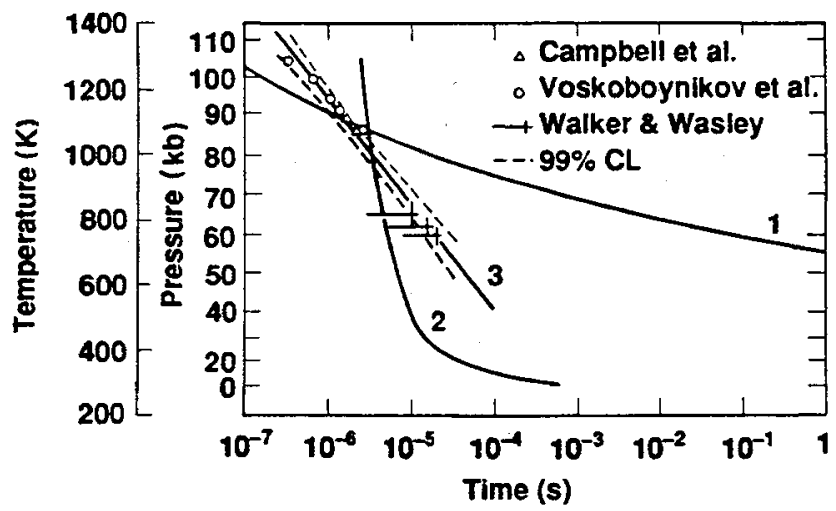

Figure 3. The calculated time to initiation as a function of shock pressure or shock temperature for a thermal equilibrium process (curve 1) compared with the initiation data for nitromethane. ${ }^{38}$ 
Acceleration of the Shock Front in Nitromethane with Non-Initiating Shocks

The results from the NM experiments just described brought into doubt the classical model of the initiation of homogeneous explosives. Strong evidence existed that some chemical reaction was started at or very near the shock front that grew rapidly close behind the front into a detonation. Also, streak-camera records provided evidence that before the detonation appeared, the shock front was being accelerated some small amount.

To explore this possibility further, we designed a series of experiments (Ref. 16) in which the velocity of non-initiating shock waves developed in ethylene glycol could be measured as the shocks passed through tanks of NM. Framing cameras showed that the shocks were accelerated above the expected hydrodynamic values as they passed through the $\mathrm{NM}$ and that the increased rate of acceleration was a direct function of the shock pressure profile. Additionally, a computercalculated model of the experiment (Fig. 4) showed that the amount of nitromethane decomposition energy (about 1 to $4 \%$ ) required to explain the experimental data, when included in the calculations, gave an excellent reproduction of the experimental results.

This is a key observation, because it indicates a level of reaction in or very near the shock front that is greater than a thermal-equilibrium process would produce. Furthermore, it shows that the shock-front acceleration occurs with no subsequent explosion or detonation in the NM to provide energy to the shock from behind it. More explicit$1 \mathrm{y}$, it says the shock front is a very narrow, nonequilibrium zone.

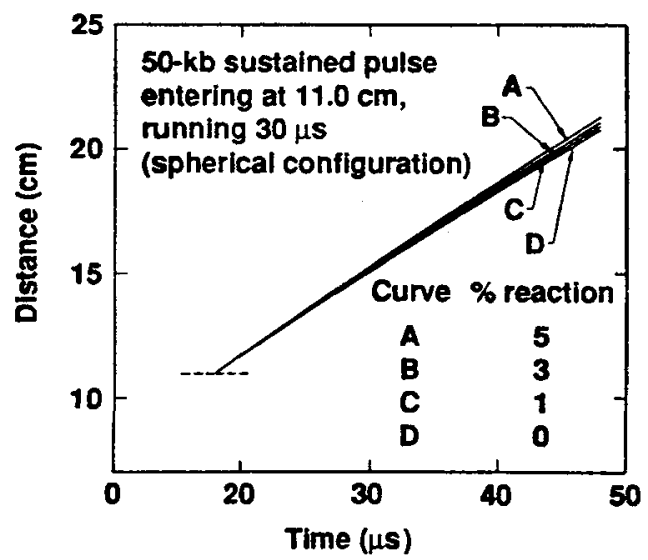

Figure 4. A computer calculation of experiments used to study acceleration of a shock front in shocked nitromethane. 
Initiation Patterns Produced in Explosives by Low-Pressure Long-Duration Shock Waves

Following the previous experiment, it seemed important to investigate the shock front and the area near it more closely to study any observable processes there. In three new experiments with low-pressure shocks ( 4.0 to $6.0 \mathrm{GPa}$ ), we designed and fired long-duration (20 to 40 microseconds) shocks. The reason for working in the lower shock-pressure regime is that the initiation zone and time are lengthened. This allows more detailed and explicit framing-camera records to be obtained.

In the first experiment of this set (see Fig. 5), a spherical shock wave produced in a large tank of water passes over two disks of IX-10 (a plastic-bonded HMX explosive). The photo record obtained by the framing camera shows that the number of initiation sites is a direct function of the shock pressure and that the sites appear quite randomly in time and space. This work was corroborated in a similar experiment by L.G. Green at the Lawrence Livermore Laboratory.

In a following experiment, in which NM was shocked just below an initiation level (Ref. 17), random centers of reaction again appeared and coalesced behind the shock front, but ahead of the face first put in compression. In all of these experiments, absolutely no evidence existed for a detonation developing at the surface first impacted.

\section{Although} the photos were so plain that no alternate explanations were seriously proposed, and although an experiment

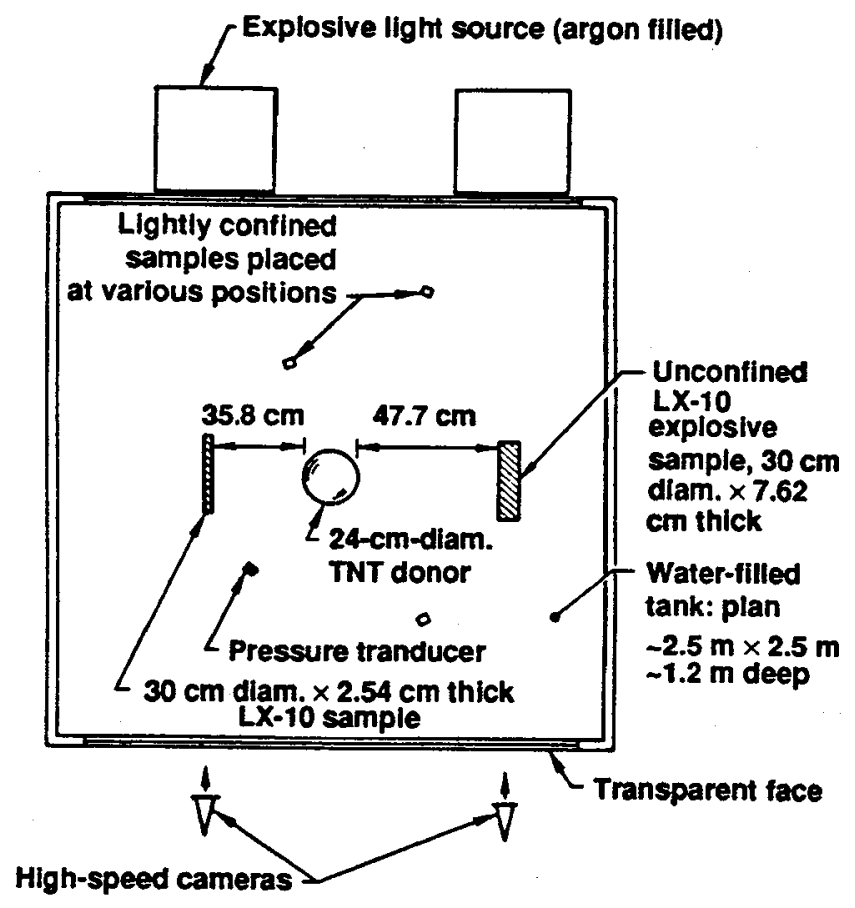

Figure 5. Design of experiment to observe effects of lowpressure shocks on LX-10. 
conducted independently corroborated the results in the solid explosives, the data were generally simply ignored because they did not fit the historic model.

Forces and Temperature in the Shock Front

Analysis of our previously described experiments and other available data led to the consideration of the possibility that mechanical fracture of some of the covalent bonds did indeed occur in and near the shock front. Experiments had been (Ref. 18) and continued to be (Ref. 19) performed in which mechanical bond scission was proposed and supported by analysis. Calculation (Ref. 2) of the probable acceleration and shear forces in and near a shock front showed that mechanical forces on atomic dimensions were likely to be stronger than the covalent bonds (i.e., C-N, N-O, C-C) in organic explosives. It seemed probable that at free surfaces of the explosive crystals, or in voids or at crystal and lattice defects, mechanical bond fracture could occur that would produce free radicals and atoms, very energetic ions, and excited molecules and molecular fragments that could react very quickly (in ps to tens of $f s$ ), and essentially in place, to produce the chemical energy that would accelerate the shock front or detonation front and maintain it.

Another facet of this study of the shock forces in and near the front is that the microscopic lattice can be distorted, and the atoms right in the front must be accelerated for at least some small distance (about 1 to 4 angstroms) up to the velocity of the shock or detonation wave by the momentum from the atoms immediately behind them. As the shock front is, in reality, the motion of these atoms in the front, they are accelerated from some random thermal motion to the velocity and with the more nearly uniaxial motion of the shock. The magnitude of the average acceleration can be calculated easily from

$\mathrm{a}=\frac{\mathrm{v}}{\mathrm{t}}$.

The force, $f=$ ma, providing this acceleration can exceed the covalent bond strength ( $R e f .2$ ), so that the explosive material (or other organics) would be fragmented at open faces, voids, and some defects. This has been corroborated in many molecular dymamics (MD) calculations, with indications that at very high shock pressures, scission can occur within the lattice and within enclosed molecules.

One other significant fact that must be considered in this context is that at the front, the major motion of the atoms is directed in one dimension, along the axis of the shock, so that temperature, normally considered as random gaussian motion of the atoms, is not a viable concept within the shock front (Ref. 20). At some distance behind the front where thermal equilibrium is again approached (tens of ps to ns) a temperature may be measured. Great efforts to measure temperature in the front have been disappointing. Estimates 
of the "one-dimensional" temperatures in various detonating explosives, based on the one-dimensional velocities along the shock axis, have ranged from 10,000 to $30,000 \mathrm{~K}$ ( $\operatorname{Ref} .20$ ).

\section{Free Radical Initiation of Gases}

P. Urtiew, E. Lee and I conducted some experiments to study the hypothesis that concentrations of free radicals could lead to detonation. In a system of silane and tetrafluorohydrazine, in which radicals formed rapidly upon mixing of the two gases, we mixed a scavenger, cis-2-butene, in the silane in sufficient concentration to keep the reaction under control until the two gases were well mixed. otherwise, reaction would have occurred immediately on contact at the gasgas interface. We thus demonstrated (Ref. 21) that the wellmixed gases would detonate once the free-radical scavenger had been consumed.

This demonstration of the production of a detonation from a high concentration of free radicals without an impacting shock was supported in experiments by J. Lee et al. (Ref. 22 ) on xenon-irradiated mixtures of hydrogen and chlorine, hydrogen and oxygen, and acetylene and oxygen. The classical theory provides no explanation for these phenomena.

In another series of experiments (Ref. 4), several chemicals known to be able to supply or capture free radicals were added to TNT at the 5 weight percent level. The impact sensitivity of the TNT in a drop hammer sudy was changed dramatically by these additives. This work was continued by impacting samples of TNT and the additives with flying plates in an air gun. The results were consistent with the drophammer results.

Inert solids and liquids, very hard and grainy materials, and some very sensitive explosives were added to the TNT in separate control studies. However, the changes in sensitivity made by the free-radical donors and getters were much greater than with any of the control additives.

Initiation and Detonation of Nitromethane with Diethylene Triamine (DETA) Added

Although amines were known to sensitize MM to shock initiation, not much quantitative data relating to this observation existed. Therefore, Wasley and I conducted a series of experiments (Ref. 23) using the same geometry shown in Fig.2, but now small amounts $(0.01$ to $5.0 \mathrm{wt} \%)$ of DETA were added to the nitromethane just before the experiments were fired. The decrease in time to initiation as a function of DETA concentration is shown in Table 2. and Fig. 6a.

Table 2 and Fig. $6 \mathrm{~b}$ also show that the detonation velocity of the nitromethane changed as a function of DETA concen- 


\begin{tabular}{|c|c|c|}
\hline $\begin{array}{c}\text { DETA } \\
\text { Conc: }(\%)\end{array}$ & $\begin{array}{l}\text { Time to } \\
\text { det. (us) }\end{array}$ & $\begin{array}{l}\text { Detonation } \\
\text { velocity }(\mathrm{mm} / \mu \mathrm{s})\end{array}$ \\
\hline 0.0 (control) & $18 \pm 2$ & $6.40^{\circ}$ \\
\hline 0.0 (control) & $\varphi^{b}$ & $6.34^{2}$ \\
\hline 0.01 & $8.4^{6}$ & $6.45^{\circ}$ \\
\hline 0.02 & $7.8^{b}$ & $6.47^{\circ}$ \\
\hline 0.05 & $7.3^{b}$ & $6.76^{2}$ \\
\hline 0.05 & $6.8^{\mathrm{b}}$ & $6.70^{\circ}$ \\
\hline 0.05 & $6.7^{\circ}$ & $6.45^{\circ}$ \\
\hline 0.10 & $5.4^{6}$ & $6.52^{2}$ \\
\hline 1.0 & $2.0 \pm 0.5$ & $6.41^{2}$ \\
\hline 5.0 & $0.5 \pm 0.3$ & $6.22^{2}$ \\
\hline $\begin{array}{l}: \pm 0.07 \\
\pm 1 .\end{array}$ & & \\
\hline
\end{tabular}

tration. This was another specific violation of the C-J, ZND, and other classical concepts. Changes in reaction kinetics were not supposed to affect detonation velocities. Further, with the use of an equation derived by skidmore and Hart (Ref. 24) that relates changes in detonation velocities to overdriving detonation pressures, the "C-J pressure" of the new nitromethane reaction with $0.05 \%$ of DETA added would appear to be near 19 $\mathrm{GPa}$. This would require a dramatic change in reaction rate. At a level of $0.05 \%$ DETA, the measured detonation velocity in three separate experiments was about $6.72 \mathrm{~km} / \mathrm{s}$, compared to the normal value of 6.32 . This would seem to require that free-radical mechanisms enhanced by the DETA be involved to give the results shown in Table 2 and Fig. 6b. The dashed line in Fig. 6b shows the detonation velocity that was calculated with the TIGER code (Ref. 25), using a selected EOS and the thermodynamic principles from the old model.

Several other interesting results were observed in this series of ten highly technical and costly experiments. As in the earlier work with neat $N M$, the origin of the detonation is at or very near the shock front in the experiments with $0.1 \%$ or less of added DETA. The retonation from the zone where the detonation originated is clearly evident. The redbrown color (probably from nitrogen dioxide), also seen by Cook (Ref. 25) in his NM initiation studies, is shown in the 
framing-camera records to sweep backwards toward the face first impacted, in consanance with the retonation. In these experiments with the lower concentrations of DETA, some initiation sites appeared at separated points at the shock front and coalesced into the detonation front, as seen in our earlier experiments with low pressure shocks.

When the DETA concentrations were $1 \%$ and $5 \%$, the detonation developed in about 2 and 0.5 microseconds, respectively. This is similar to the time to detonation in NM with no additives with shock pressures of about 7.5 to $9.0 \mathrm{GPa}$, compared to the $6.0 \mathrm{GPa}$ in these experiments. An interesting phenomenon seen in the framing-camera photos of these two firings was that the initiation occurred in hundreds of small centers of reaction, which quickly coalesced into the detonation front. The small centers appeared first to be distributed quite randomly in time and space. The pattern was finer at the higher concentration. This is remiscent of the results seen previously in the initiation studies of heterogeneous explosives (Ref. 17), except that here the number of reaction sites is a function of increased DETA concentration rather than increased pressure. However, both increased DETA concentration and increased pressure lower the time to detonation.

Here is another observation of considerable interest. Now that the detonation occurs in about 1 microsecond, as in the earlier work (Ref. 6) at $8.0 \mathrm{GPa}$, the detonation appears in the streak-camera photos to originate at the nitromethane face first impacted. However, it can be seen easily in the framing-camera photos that the detonation is actually forming in the narrow band where the reaction sites are coalescing. Thus, in the old streak records (Ref. 6), the detonation would have appeared to come from the container-NM interface. In fact, in some of those early records, the reaction light seems to reach rather tenuously toward the interface. This same phenomenon would have made it difficult for Hardesty (Ref. 26) to observe the exact position of the detonation front in his initiation study. Recent experiments (Ref. 25) on the initiation of liquid nitric oxide support the contention that the detonation is formed in a narrow zone where the reaction sites are coalescing and not at the containerexplosive interface.

The BTNEA Experiment

A homogeneous ideal explosive, bis-trinitroethyl adipate (BTNEA) was synthesized with the isotopic labels (carbon thirteen and oxygen eighteen) introduced into the positions indicated in Fig. 7. This explosive was chosen for this experiment, because it appeared that the $C O$ and carbon dioxide molecules expected as detonation products were already formed, and the isotopic labels would be found in the $C O$ and carbon dioxide products. 


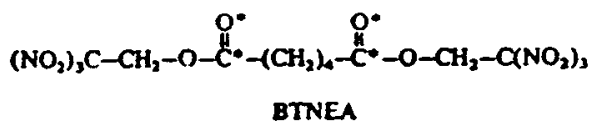

Figure ?. The structure of bis-triniurcethyl adipate. The asterisks indicate carbon atoms of isotope 13 and oxygen atoms of isotope 18 .

The explosive was detonated in a bomb calorimeter in which the products were collected and then analyzed for the isotopic ratios. (Ref. 27). The experimental results show that the ratios of $\mathrm{c}^{12} / \mathrm{C}^{13}$ and $0^{16} / \mathrm{o}^{18}$ are essentially the same for all of the products containing $C$ and/or 0 , and they are nearly equal to the isotopic ratios in the original BTNEA sample. The analytical values of the ratios were said to be weli within the experimental precision of the determination. The conclusion that is obvious is that almost every covalent bond was broken, the atoms were scrambled, and they were randomly combined into the detonation products. Quoting from the paper. "We must conclude that, in the case of the homogeneous ideal explosive, all of the bonds of the original explosive molecules are, in effect, broken during the detonation process. These molecular fragments then must recombine in a statistically random fashion prior to the kinetic "freeze out" of products during the adiabatic expansion. Certainly, diffusion on a molecular level cannot be an important ratecontrolling process."

Is Detonation Velocity Determined by Thermodynamics or Atomic Vibrational Velocities?

From the early days of the study of explosives, detonation velocities have been known to be relatively constant for a given explosive. In the ZND and other hydrodynamic models, the major velocity determinant is considered to be the thermodynamic content of the explosive, which provides a definitive pressure during the detonation. Reaction kinetics are considered to be irrelevant to the process.

The NM-DETA experiments previously discussed indicate that kinetics may affect detonation velocities. However, as seen in the DETA work (Ref. 23), even with an almost 50\% increase in detonation pressure, the detonation velocity of NM increased only about 6\%. It appears obvious that there is some large energy loss or some restraining factor. Is there some process that controls the detonation's reaction rate in a chemical or physical sense that was not previously considered?

Many recent quantum-mechanical and other (Ref. 25) kinetics studies have supported the contention that the shocked system is not in thermal ewuilibrium, principally 
because the calculations show that the acoustic energy from shock waves is transferred too slowly to a thermally equilibrated state of the intramolecular vibrons. These results support the concept of the nonequilibrium nature of the shockfront processes (Ref. 4), but they do not help much to explain what is occurring at or very near the shock front to cause it to accelerate, what causes the reaction patterns seen, and what causes the appearance of the detonation at or very near the front; nor does it tell us why detonation velocities should have the values they do or why they should be relativeiy constant. These questions will be addressed below.

The scale of $\mathrm{km} / \mathrm{s}$ in which shock or detonation velocities are usually given is the same scale as angstroms per $\mathbf{s}^{-13}$. The significance of this observation is that during shock initiation or detonation, the front is moving across a covalent bond of an explosive in a period on the order of the vibrational frequency. When one calculates the relative velocity of the vibrating atoms in a $\mathrm{C}, \mathrm{H}, \mathrm{O}, \mathrm{N}$ system by three different methods (Ref. 25), these velocities are found to fall in the same magnitude as the shock and detonation velocities. Could this be the key to the velocity restraint and the stabilization of detonation velocities?

In MD calculations of covalent systems (see Ref. 25 for the references to studies reported in this paragraph) related to organic explosives, John Hardy, Arnold Karo and I found a shock front to be quite narrow (about 10 to 100 angstroms). This same result was obtained in MD calculations by Dremin (Ref. 20) and Holian; in MD calculations of detonating systems by Peyrard et al., Iambrakos et al., and Elert et al.; in quantum mechanical (QM) calculations by Coffey and Toton, Dancz and Rice, Zerilii and others; in light-reflection experiments in NM and water by Harris and Presles, and Kormer, Campillo et al., and others. If this is so, then the shock front energy is held in a very narrow band, and the energyor momentum-transfer rate is enormous, as was calculated by us previously (Ref. 5). This suggests that the acceleration and shear forces in the shock front are of the magnitude previously calculated ( $\operatorname{Ref} .2$ ), which are of the order to mechanically scission covalent bonds, particularly at voids, surfaces, crystal defects, etc.

If the Hugoniot curves of a number of organic materials are compared for the pressure range of 0.2 to $30 \mathrm{GPa}$, the shock velocities all fall quite close, running from about 3 to 9 $\mathrm{km} / \mathrm{s}$. The unreactive Hugoniots (no chemical energy released) of the:organic explosives are very nearly the same. Now, if the detonation pressures and velocities of most of the common explosives are plotted on the same graph with these other values, they fall very near this shock velocity curve of the inert or unreacted materials (Ref. 3 and Fig. 8). 
If a small allowance (about $10 \%$ ) is made for the addition to the shock velocities due to the much higher temperatures in the detonating explosives, all of the $D$ values of these 15 commonly-used and studied explosives are enclosed in this small space between the Hugoniot shown for the inert materials, or the unreacted curve for triaminotrinitrobenzene (TATB), and the $10 \%$ increase line. This means that the extremely rapid release of the great quantities of chemical energy in the detonating explosives has only a relatively small effect on the shock or detonation velocity (D). The piston formalism denies the importance of and excludes kinetics from consideration. However, since the Ds are indeed relatively constant for a specific material, a physical or chemical explanation is required:

Remembering that

shock fronts cross the interatomic bonds of organic materials in times of the same order as the vibration periods (Ref. 28), I attempted to calculate the relative vibrational velocities of the atoms of these bonds using three distinct methods: (1) infrared and $x$-ray crystallographic data (Ref. 28), (2) MD calculations (Ref. 25), and (3) the HulburtHirschfelder equations (Ref. 25). It was seen that the detonation velocities for all of the organic explosives lie in the band of vibration velocities characteristic of the $\mathrm{C}, \mathrm{H}, \mathrm{O}, \mathrm{N}$ atom pairs (Ref. 28). Additionally, we found that the relative vibrational velocities rise slowly to moderate maxima even at extremely high temperatures.

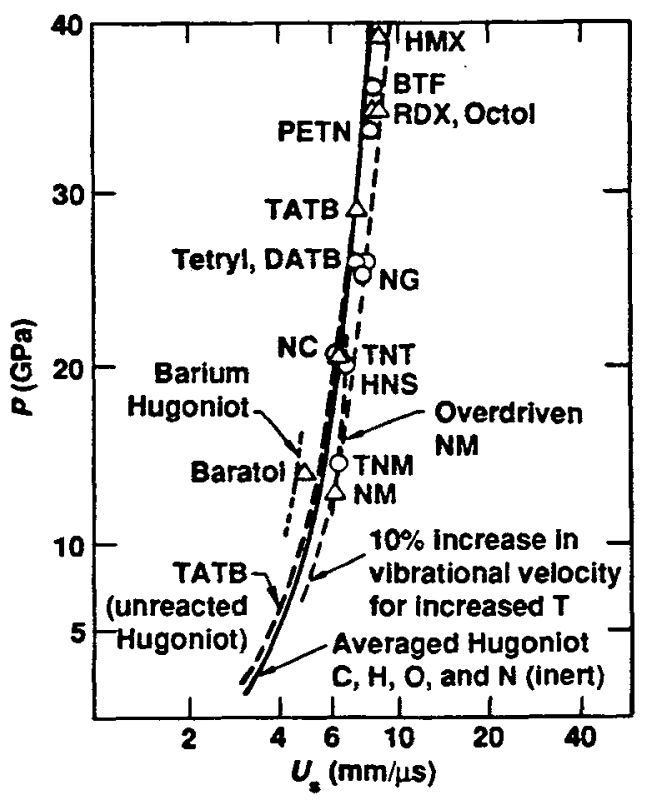

Figure 8. Comparison of Hugoniot curves for organic materials with the detonation velocities of common explosives.

A rather simple physical explanation exists, then, for a near constancy of detonation velocities. The vibrational motion that carries the principal bond-scission activating energy can proceed through the detonating explosive, even at very high temperatures, only at or near the relative vibrational velocities. That is why greatly increased levels of shock pressure and high temperatures add little to detonation velocities. 
Thus, as shown in Ref. 23, kinetics is important and can change detonation velocities, but a large increase in reaction rate (or energy release rate) and high additional pressure (Ref. 24) make only a small increase in the D. This does not mean that thermodynamic energy content does not influence detonation velocities or explosive power output. It does mean, however, that it is not the thermodynamic energy content that restrains the velocities to a range of about 5 to $9 \mathrm{~km} / \mathrm{s}$.

Another observation (Ref. 29) on detonation that adds concerm about a purely thermodynamic constraint is that Ds measured along different crystal axes in single crystals of RDX and PETN have different values. This can be explained in the new theory as a result of different kinetics due to different free radicals and molecular fragments and ion species formed by the mechanical forces on the different molecular orientations in the crystal lattices.

\section{SIGNIFICANT MOLECULAR DYNAMICS CALCULATIONS}

Two-Dimensional Calculations of the Effects of Lattice Defects

The macroscopic effects of the increase in sensitivity to shock initiation caused by crystal defects such as voids or cracks or very irregular crystal structure or the inclusion of heavy particles in an explosive had been experimentally observed. However, no calculations on the atomic scale had been found that simulated these conditions. Therefore, we completed a series of two-dimensional (2D) MD calculations to study these conditions. In every case, the defect studied showed a substantial increase in the number of bond scissions and energy concentrations at the sites of the defects (Ref. 25).

We next introduced a mathematical concept by which an amount of energy equal to approximately the heat of detonation per bond was added to the calculation along a reaction coordinate where bond scission had occurred. The idea considered was that the radicals formed from the endothermic bond fracture would react in about $10^{-13} \mathrm{~s}$, thereby adding exothermic reaction energy to the system not far from where the scission occurred, when the radicals reacted. We made a number of calculations in different geometries and at different initial temperatures using this "reactive" potential. The effect was dramatic (Ref. 3). This study illuminated another factor-the time scales involved in the different initiation models that had been proposed. The mechanical bond scission could lead to exothermic reaction at times on the order of $10^{-13} \mathrm{~s}$, and this could, therefore, influence the shock velocity by providing significant reaction energy at or very near the shock front. On the other hand, the equilibrium thermal processes proposed (gas compression, friction heating, etc.) all require much longer times (on the order of nanoseconds 
to microseconds) to provide significant exothermic response. The shock front in these cases would be far downstream by such times.

The shock energy in both the 1-D and 2-D calculations did not couple well with the thermal or vibrational energy in the lattices. This supported the conclusion stated earlier that "the energy in the shock front is highly nonergodic and that thermal equilibrium, particularly between the translational and vibrational energy modes, does not exist in the front."

We completed several series of 2-D and some 3-D MD calculations to explore the factors involved in shock rise times and the associated shock-front widths. In both the 2-D and 3-D studies, the shock energy stayed localized in some small number of layers ( 4 to 15 ) of atoms. In calculations with the initial vibrational motion of the lattice atoms simulating conditions near the melting point or cold (with no initial thermal motion), the net results as to the shockfront widths and thus the rise times were similar (Ref. 25).

If one considers the case in which the shock front stays coherent in 12 layers of atoms (about 24 angstroms) and an initiating shock was proceeding into the material at 4 angstroms in $10^{-13} \mathrm{~s}(4 \mathrm{~km} / \mathrm{s})$ the microscopic rise time would be $6 \times 10^{-13} \mathrm{~s}$. In the case of a detonation front moving at 8 angstroms in $10^{-13} \mathrm{~s}$, the microscopic rise time is $3 \times 10^{-13} \mathrm{~s}$. Allowing for some lattice irregularities and slightly increased intermolecular distances in real systems, rise times could be near picoseconds, and the shock width would be close to the span of 15 water molecules, as proposed by Harris and Presles (Ref. 30).

\section{Calculations of Shock-Induced Chemistry}

A new field that combines physics and chemistry has come into prominence in the past decade. This research and development involves the synthesis and fabrication of new compounds, alloys, and other materials through shock-induced chemical and physical reaction (Ref. 25). The chemistry and physics in these processes are directly related to the shockinduced reaction proposed in the new theory.

To add more realism to the MD calculations, we made two diatomic studies in which nitric oxide and sulfur nitride lattices were simulated: in the first case we calculated the effect of the impact of an aluminum plate on a model facecentered-cubic nitric oxide lattice containing a void; and in the second case, a similar geometry was used in which the aluminum plate impacted a model face-centered-cubic sulfur nitride lattice. The calculations were made in each case with the initial random motion of the atoms representative of room 
temperature and again when the systems were "cold," with no initial thermal motion.

These four sets of calculations were compared with those of an earlier, more general, covalent system. The same qualitative results were obtained of bond scission, with atoms and molecular fragments flying across the void to impact the opposite walls and cause further scission. When the reactive potential was used in a similar geometry in a more general lattice, the initial "spall" from the inner surface of the void and the impact on the opposite void face led to activity of the atoms that was very suggestive of hot spot formation through free-radical chemistry. These motions and simulated reactions are all nonergodic, nonequilibrium processes.

\section{SUNMARY OF MORE RECENT SUPPORTIVE EVIDENCE}

Sharma et al. reported (Ref. 31) studies of initiation sites found in TATB shocked to near-initiation levels. They found the sites, by scanning electron microscopy (SEM), to be tiny holes on the surfaces and edges of the explosive grains. They showed, by $x$-ray photoelectron spectroscopy (XPS), that deposits of acetone-soluble reaction products in the holes were furoxan and furazan derivatives of TATB. Their analysis suggested that the furoxan product could react with adjacent $T A T B$ molecules in an exothermic chain reaction to give a water molecule and a new furoxan. Thus the shock=formed furoxan could immediately (i.e., in $10^{-13}$ to $10^{-12} \mathrm{~s}$ ) provide reaction energy very near the shock front. Sharma suggested that this reaction could be involved in the initiation of TNT as well.

Tanaka et al. provide a strong defense of the new theory. They report (Ref. 32) that an explosive designated as E25 (75\% PETN $/ 25 \%$ paraffin) at a density of $1.265 \mathrm{~g} / \mathrm{cc}$ has a measured $D$ of $7.230 \mathrm{~km} / \mathrm{s}$, whereas pure PETN (pentaerythritol tetranitrate) at the same density has a measured $D$ of $6.60 \mathrm{~km} / \mathrm{s}$. However, the calculation using shock velocities and the empirical formula gave a value of $7.267 \mathrm{~km} / \mathrm{s}$, within $0.51 \%$ of the measured value. This is well within the precision of D measurements. The classical theory calculation missed the measured value by $14.24 \%$. The author of the paper who reported the E25 data stated that, "All equations-of-state available to us cannot reproduce these results."

In 1992, Brenner, Robertson et al. published results of their MD studies in which they use many-body interatomic potentials to provide more realism to their calculations. Their excellent graphics show in unmistakable detail (Fig. 9) the narrow shock and detonation zones, the massive mechanical fracture of the covalent bonds, the free atoms and molecular fragments, and the free-radical chemistry in and very near the front. These processes are nonequilibrium and nonthermal. 


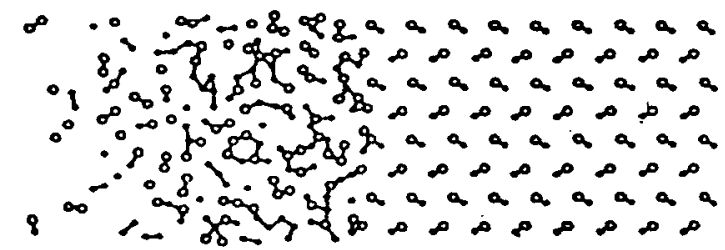

Snapshot from a simulation of a deconating film. The length of the oystein xlenwn is $\approx 60 \mathrm{~K}$

Figure9. Molecular dynamics calculation of a model detonating solid with two types of atoms and with exothermic reactivity incorporated into the dynamics.

Simpson, Helm and Kury (Ref. 34) studied the nonreactive Hugoniot for HMX/water mixtures, and they reported that with shocks of 5.17 to $5.99 \mathrm{GPa}$ there was no evidence of HMX reaction. However, they showed by comparison with these results that in solvent-pressed HMX in wedge tests about 3 to $7 \%$ of the HMX had reacted in less than about $100 \mathrm{~ns}$. They report, "The observed higher shock velocities in the solvent-pressed data we attribute to a reaction-supported shock front." other pertinent comments from this paper are: "Implicit in the use of a detonation product EOS is the assumption that chemical reactions occurring under the shock loading conditions of the wedge tests go to completion. Therefore, since early time reactions may only proceed to intermediate states, the extent of reaction inferred through reactive modeling will be conservative." "The greatest uncertainty in determination of state behavior of HMX from measurements on a mixture is the assumption of a one-dimensional shock wave passing through a homogeneous medium."

The information in these four references explicitly adds strong support for the new Walker, Wasley, Karo (WWK) theory.

NEW EQUATIONS USED TO CAICULATE DETONATION VELOCITIES

In 1968, Kamlet and Jacobs (Ref. 35) reported the development of two empirical equations for calculating detonation pressures and velocities. With some simplification of their concept and an algebraic derivation, a simple equation for calculating $D=f(P)$ was obtained:

$D=2.45+\frac{P^{\frac{1}{2}}}{3}$.

When the detonation data for 14 very different explosives are compared by means of this relationship, two interesting results appear: 
(1) The aliphatic compounds are all on one side of an averaged curve, and the aromatics (plus sterically-hindered PETN) are all on the other side.

(2) With a small positive correction for hydrogen and nitrogen content (to compensate for their relatively higher shock velocities at a given pressure) and a small off'set for aromaticity, an equation was formulated that reproduces the data for the 14 explosives within +1.57 and $-1.03 \%$. That equation follows:

$\mathrm{D}=\left(\frac{\mathrm{p}^{\frac{1}{2}}}{3}+2.0+0.25 \mathrm{a}\right)+0.05\left(\mathrm{H}_{\mathrm{p}}+\frac{\mathrm{N}_{\mathrm{p}}}{20}\right)$,

where $a=0$ if the compound is aromatic and $a=1$ if it is aliphatic, and $\mathrm{H}_{\mathrm{p}}$ and $\mathrm{N}_{\mathrm{p}}$ are the weight percents of hydrogen and nitrogen, respectively.

The concept underlying the equation is that detonation velocity is principally a rather simple Hugoniot relationship, $D=f(P)$. More explicitly, thermodynamic factors, the EOSs, and even reaction rates have limited influence on the actual values of $D$.

Kamlet's empirical equation for calculating detonation pressures is probably as useful as the TIGER thermodynamic code with the complex EOSs that are used, and we have shown that the Ds for 48 very different explosives can be calculated accurately (well within the experimental error of the better measured values) from the Hugoniot values of the elements that make up the explosives. The equation used is as follows:

$\left.D=T_{c} \sum U_{s i} f_{i}\right)$,

where $\mathrm{U}_{\mathrm{si}}$ is the shock velocity of the elements at $\mathrm{P}_{\mathrm{C}-\mathrm{J}}$, $f_{i}$ is the atomic weight fraction of the element, and $T_{c}$ is a small correction (about 3 to $8 \%$ ) (Ref. 25 ) required because the detonation temperatures are considered to be about 2500 to $5000 \mathrm{~K}$, whereas the Hugoniot values of the elements are usually measured between about 70 and $950 \mathrm{~K}$. The Ds for 21 of the best-characterized explosives were calculated with Eq. 5, and the correlation coefficient obtained for these values is 0.976 . D values were calculated for 25 other explosives for which less data were available, and the correlation coefficient for this set is 0.932, (Ref. 36).

\section{CONCIUSIONS}

It is concluded: (1) That the new concept of physical kinetics is a valid concept for determining reaction rates in detonations and in highly shocked systems. Shock and detonation velocities are related directly to the average relative vibrational velocities of the atom pairs in $\mathrm{C}, \mathrm{H}, \mathrm{O}, \mathrm{N}$ materials. 
(2) That the exceedingly high kinetic energy in the detonation front is sufficient to cause massive fracture of the covalent bonds of the molecules of explosives (and other organics) at and near the front, so that the large majority of the molecules are broken to individual atoms or radicals and are rearranged extensively, and that the rates of the subsequent very rapid chemical reactions can be influenced by the addition in the explosives of chemicals providing enhancing or inhibiting reactions.

(3) That the simple Eq. 5 is a rational equation, based on appropriate Hugoniot principles, which provides for very accurate calculation of detonation velocities from the shock velocities of the elements in the empirical formulae of the explosives.

(4) That the concepts of physical kinetics and the small increase in vibrational velocities with increasing temperature provide the earlier missing pieces that now explain the relative constancy of detonation velocities.

(5) That the comparison presented herein shows that this new modern theory (the WWK theory) of the initiation and detonation of explosives provides a realistic microscopic description of and significant utility in understanding and calculating explosives phenomena.

\section{REFERENCES}

1. W.C. Davis, Sci. Am. 256(5), 106 (1987).

2. F.E. Walker and R.J. Wasley, Propellants and Explosives 1, 73(1976).

3. F.E. Walker, Proceedings of the 19th International Pyrotechnics Seminar, 20-25 February 1994, pp. 297-318, South Pacific Inform. Services Ltd, Christchurch, N.z. (1994).

4. F.E. Walker, Propellants, Explosives, Pyrotechnics 7, 2(1982).

5. F.E. Walker, J. Appl. Phys. 63(11), 5548-5554 (1988).

6. A.W. CampbelI, W.C. Davis, and J.R. Travis, Phys. Fluids 4, 498 (1961).

7. A.W. Campbell, W.C. Davis, J.B. Ramsay, and J.R. Travis, Physics Fluids 4, 511 (1961).

8. F.P. Bowden and O.A Gurton, Nature 161, 348 (1948).

9. A. Joffe, Nature 161, 349 (1948).

10. E.F. Gittings, Fourth Symposium on Detonation (Preprints) VoI. II, C-15 (U.S. GPO, Washington, D.C., 1965).

11. H. Eyring, R.E. Powell, G.E. Duffey, and R.B. Parlin, Chem. Rev. 45, 69 (1949).

12. H. Eyring and An-Lu Leu, Proc. Nat. Acad. Sci. USA 72(5), $1717(1975)$.

13. H. Eyring, Science 199, 740 (1978).

14. F.E. Walker and R.J. Wasley, Explosivstoffe 17, 9 (1969). 
15. H. Cheung, A. Weston, L. Green, and E. James, Explosive Initiation, Lawrence Livermore National Laboratoṛy, Livermore, CA, UCRL-76578 (1975).

16. F.E. Walker and R.J. Wasley, Combust. Flame 15, 233 (1970).

17. F.E. Walker and R.J. Wasley, Combust. Flame 22, 53 $(1974)$.

18. W. Taylor and A. Weale, Trans. Faraday Soc. 34, 995 (1938).

19. M. Held, Explosivstoffe 11/12, 241 (1969).

20. A.N. Dremin and V. Yu. Klimenko, Progress in Astronautics and Aeronautics 75, J. Ray Bowen, N. Manson, A.K. oppenheim, and R.I. Soloukhin, Eds. (AIAA, New York, NY, 1981). pp.153-168.

21. P.A. Urtiew, E.L. Lee, and F.E. Walker, Arch. Thermodyn. Combust. 9, 259 (1978).

22. J.H. Lee, R. Knystautos, and N. Yoshikawa, Acta Astronaut. 5, 971 (1978).

23. F.E. Walker, Acta Astronaut. 6, 807 (1979).

24. I.C. Skidmore and S. Hart, Proc. 4th Symp. (Internat.) Detonation, U.S. Naval ordnance Laboratory, White Oak, MD, 12-15 October 1965 (U.S. GPO, Washington, D.C.) p.47.

25. F.E. Walker, Initiation and Detonation of Explosives-an Alternative Concept, Lawrence Livermore National Laboratory, Iivermore, CA, UCRL-53860, 11 January 1988.

26. D.R. Hardesty, Combust. Flame 27, 229 (1976).

27. R.R. McGuire and D.I. Ornellas, Propellants and Explosives 4,23 (1979).

28. F.E. Walker, Propellants and Explosives 6, 15 (1981).

29. H.W. Koch and Ch. Baras, Institut Franco-Allemand de Recherches de Saint-Louis, France, Rapport 28/71 (1971).

30. P. Harris and H.-N. Presles, J. Chem. Phys. $80(1), 524$ $(1984)$.

31. J. Sharma, J.W. Forbes, C.S. Coffey, and T.P. Liddiard, J. Phys. Chem. 91, 5139 (1987).

32. K. Tanaka, S. Oinuma, et al., Shock Compression of Condensed Matter 1989, S.C. Schmidt, J.N. Johnson, L.W. Davison, (editors), Elsevier Science Publishers B.V., (1990).

33. D.W. Brenner, D.H. Robertson, et al., Microscopic Simulations of Complex Hydrodynamic Phenomena, Edited by M. Mareschal and B.I. Holian, Plenum Press, New York, NY, pp. 111-123 (1992).

34. R.I. Simpson, F.H. Helm, and J.W. Kury, Propellants, Explosives, Pyrotechnics 18, 150-154 (1993).

35. M.J. Kamlet and S. Jacobs, J. Chem. Phys. 48(1), 23 (1968).

36. F.E. Walker, Propellants, Explosives, Pyrotechnics 15, $157-160$ (1990). 
Table 3. Correlations Between Reaction Dynamics Experiments and Detonation Energy and Time Characteristics.

Property

Velocities of atoms and molecules

Center of mass. $(I \cdots C N) *$

Translation of $\mathrm{H}$ atoms

Velocities of atoms and molecules

in detonation front

" $\mathrm{C}$ " and " $\mathrm{H}$ " atoms from $\mathrm{MD}$ calcs.

Vibrational velocities of atom

pairs as $f(T)$ at second vib.

level--from QM calculations**

$\mathrm{H}$ atoms from detonating charge

Energy of atoms and molecules

For the reaction:

$$
(I-C N)-(I \cdots C N) *-I+C N
$$

(Energy available for reaction)

$\mathrm{H}+\mathrm{OCO}-(\mathrm{H} \cdot \mathrm{OCO}) *-\mathrm{OH}+\mathrm{CO}$

( $H$ kinetic energy)

Energy of PES barrier to TS

Kinetic energy of atoms at $8 \mathrm{~km} / \mathrm{s}$

$\mathrm{N}$

0

Bond energies in $\mathrm{RDX}$

$\mathrm{C}-\mathrm{N}$

$\mathrm{N}-\mathrm{N}$

Times to reaction

Iifetime of TS
$(I . . \mathrm{CN}) *$
$0.2 \mathrm{ps}$
$(\mathrm{H} . \mathrm{OOCO})^{*}$
$5.0 \mathrm{ps}$

\section{Reaction Dynamics Detonation}

$20 \mathrm{~km} / \mathrm{s}$

6 to $9 \mathrm{~km} / \mathrm{s}$

1 to $20 \mathrm{~km} / \mathrm{s}$

$\mathrm{HH} \quad 13.7 \mathrm{~km} / \mathrm{s}$

$$
\begin{array}{ll}
\mathrm{CH}, \mathrm{NH}, \mathrm{OH} \cdot & 8.1 \text { to } 9.6 \mathrm{~km} / \mathrm{s} \\
\mathrm{CC}, \mathrm{NN}, 00 & 2.0 \text { to } 2.7 \mathrm{~km} / \mathrm{s} \\
& 15 \mathrm{~km} / \mathrm{s}
\end{array}
$$

$0.883 \mathrm{eV}$

$2.07 \mathrm{eV}$

$105 \mathrm{~kJ} / \mathrm{mol}$
$4.72 \mathrm{eV}$

$5.34 \mathrm{eV}$

$2.30 \mathrm{eV}$

$1.06 \mathrm{ev}$ 
Table 3. (cont.)

From MD calculations

"Time to reaction"

$\mathrm{C}-\mathrm{H}$ in " $\mathrm{CH}_{2}$ "

0.06 to $0.24 \mathrm{ps}$

$\mathrm{C}-\mathrm{H}$ in "PETN" interior

0.6 to $1.0 \mathrm{ps}$

$\mathrm{N}-\mathrm{O}$ in "PETN" interior

0.4 to $1.0 \mathrm{ps}$

$C-C$ in " $C$ " matrix

0.06 to $0.3 \mathrm{ps}$

Estimate of time to reaction

in detonation at $8 \mathrm{~km} / \mathrm{s}$

In 20 angstrom zone

$0.25 \mathrm{ps}$

In 40 angstrom zone

$0.5 \mathrm{ps}$

* From Hulbert-Hirschfelder calculations 